\title{
A CRISPR screen for factors regulating SAMHD1 degradation identifies IFITMs as potent inhibitors of lentiviral particle delivery
}

\author{
Ferdinand Roesch, Molly OhAinle and Michael Emerman * (1)
}

\begin{abstract}
The InterFeron Induced TransMembrane (IFITM) proteins are interferon stimulated genes that restrict many viruses, including HIV-1. SAMHD1 is another restriction factor blocking replication of HIV-1 and other viruses. Some lentiviruses evolved VpxNpr proteins to degrade SAMHD1. However, this viral antagonism can be perturbed by host mechanisms: a recent study showed that in interferon (IFN) treated THP1 cells, Vpx is unable to degrade SAMHD1. In the present work, we designed an Interferon Stimulated Genes (ISGs)-targeted CRISPR knockout screen in order to identify ISGs regulating this phenotype. We found that IFITM proteins contribute to the IFNa-mediated protection of SAMHD1 by blocking VSV-G-mediated entry of the lentiviral particles delivering Vpx. Consistent with this, IFNa treatment and IFITM expression had no effect when the A-MLV envelope was used for pseudotyping. Using an assay measuring viral entry, we show that IFNa and IFITMs directly block the delivery of Vpx into cells by inhibiting VSV-G viral fusion. Strikingly, the VSV-G envelope was significantly more sensitive to this IFNa entry block and to IFITMs than HIV-1's natural envelope. This highlights important differences between VSV-G pseudotyped and wild-type HIV-1, in particular relative to the pathways they use for viral entry, suggesting that HIV-1 may have evolved to escape restriction factors blocking entry.
\end{abstract}

Keywords: HIV-1, SAMHD1, Vpx, Pseudotypes, Interferon, IFITM, VSV-G, A-MLV, Viral entry

\section{Background}

Type I IFNs play a central role in activation of innate immunity by turning on a vast transcriptional program that results in enhanced expression of hundreds of Interferon Stimulated Genes (ISGs). Many of these ISGs encode proteins that have antiviral activity, inhibiting viruses at various steps of their life cycle including at the earliest stages of entry [1]. In particular, the InterFeron Induced TransMembrane (IFITM) proteins (IFITM1, IFITM2, and IFITM3) are ISGs localized at the cell surface and in endosomal compartments that restrict entry of many viruses, including HIV-1, VSV, Influenza A, some flaviviruses and alphaviruses [2-6].

*Correspondence: memerman@fredhutch.org Divisions of Human Biology and Basic Sciences, Fred Hutchinson Cancer Research Center, 1100 Fairview Ave N, Mailstop C2-023, Seattle, WA 98109, USA
After fusion, retroviruses release genomes and associated proteins into the cytoplasm during the uncoating process, ultimately resulting in the synthesis of reverse transcription products, formation of the preintegration complex, and nuclear import [7]. The accessory proteins $\mathrm{Vpr}$ and $\mathrm{Vpx}$, which are encapsidated into budding virions through interaction with the p6 region of Gag [8], are also released from the incoming viral particles after fusion, in a process independent from uncoating of the viral core [9].

One of the functions of $\mathrm{Vpx}$ is to promote degradation of the host antiviral protein SAMHD1 [10, 11]. SAMHD1 is a dNTP triphosphohydrolase that depletes the cellular pools of dNTPs [12] in quiescent cells of the myeloid lineage and in resting $\mathrm{CD} 4+\mathrm{T}$ cells [13], resulting in a block to reverse transcription $[10,11]$. In some SIVs lacking Vpx, SAMHD1 antagonism is achieved by the related viral protein $\mathrm{Vpr}$ [14]. Vpx/Vpr proteins directly bind to 
SAMHD1 and recruit the DDB1/Cul4/DCAF1 ubiquitin ligase complex, resulting in proteasomal degradation of SAMHD1 $[15,16]$. The recognition of SAMHD1 by Vpx/ Vpr proteins is evolutionarily dynamic, with some viral proteins binding to the $\mathrm{N}$-terminus of SAMHD1, and others to the C-terminus [17]. In addition to the evolution at the Vpx/SAMHD1 interface, the host has evolved other mechanisms to regulate SAMHD1. For instance, SAMHD1 enzymatic activity is controlled by cell cycle progression [18-20], and the host factor H11/HSPB8 has been reported to degrade Vpx, thus protecting SAMHD1 from degradation [21].

Dragin et al. [22] showed that interferon alpha (IFN $\alpha$ ) treatment of THP1 cells prevents degradation of SAMHD1 following incubation with SIV $_{\text {MAC }}$ virus-like particles containing Vpx (VLPs-Vpx). This observation suggests the existence of one or more proteins induced or activated by IFN $\alpha$ that directly or indirectly protect SAMHD1 from degradation. IFN treatment controls SAMHD1 enzymatic activity by phosphorylation [18], and reports have described induction of SAMHD1 expression in monocytes and some cell lines after IFN $\alpha$ treatment $[23,24]$ through mechanisms involving modulation of microRNAs and IRF3, respectively. However, none of these effects could explain how IFNa protects SAMHD1 from degradation by Vpx [22].

In the present work, we confirmed that treatment of THP1 cells with either interferon alpha (IFN $)$ or interferon gamma (IFNY) indeed protects SAMHD1 from degradation by Vpx when Vpx is delivered to cells via VLPs that are pseudotyped with VSV-G. We designed a flow cytometry-based CRISPR knockout screen to identify ISGs involved in this phenotype and identified the InterFeron Induced TransMembrane (IFITM) proteins. Moreover, we show that IFNa protects SAMHD1 from degradation when Vpx-containing VLPs are pseudotyped with VSV-G for viral entry, but not when they are pseudotyped with the envelope from amphotropic murine leukemia virus (A-MLV), suggesting that the IFN $\alpha$ block occurs at the level of viral entry. By directly comparing the entry block imposed by IFN $\alpha$ and IFITMs on HIV-1 pseudotyped with different envelopes, we show that VSV-G is inhibited to a greater degree than HIV-1 wild-type envelope. This result suggests that HIV-1 may escape IFN $\alpha$ induced blocks to viral entry, potentially by using different cellular pathways for membrane fusion, as suggested in a recent study [25]. Finally, our screen could also be used to identify factors blocking a variety of different viral envelopes, using SAMHD1 degradation as a proxy for cytoplasmic delivery of viral proteins.

\section{Results \\ IFN treatment protects SAMHD1 from degradation by Vpx in THP1 cells}

First, we confirmed the published observation that IFN treatment inhibited SAMHD1 degradation in THP1 cells [22]. SAMHD1 degradation in THP1 cells was achieved by overnight treatment with Virus-Like Particles (VLPs) that consist of the $\mathrm{SIV}_{\mathrm{MAC}} \mathrm{Gag} /$ Pol structural proteins and naturally package both Vpr and Vpx [26], with only Vpx being able to degrade SAMHD1 [14]. Vpx-containing VLPs (VLPs-Vpx) were pseudotyped with the Vesicular Stomatitis Virus glycoprotein (VSV-G), as has been done in previous studies $[10,12,22,27]$. THP1 cells were treated with universal type I interferon alpha (IFNo), a recombinant human IFN alpha, or IFN $\gamma$ for $24 \mathrm{~h}$ and transduced with VLPs-Vpx. We measured the endogenous levels of SAMHD1 using flow cytometry in order to monitor SAMHD1 degradation in a quantitative manner in cell populations. Over $99 \%$ of the cells expressed SAMHD1, and this proportion did not change with IFN $\alpha$ or IFN $\gamma$ treatment (Fig. 1a, top row). Consistent with previously published data, SAMHD1 was degraded in the presence of VLPs-Vpx (Fig. 1a, bottom row) and high levels of SAMHD1 degradation can be achieved: it reached $50 \%$ in the dose response presented in Fig. $1 \mathrm{~b}$ (black lines), and even higher levels if more VLPs-Vpx were used (not shown). However, in the presence of IFN $\alpha$ and IFN $\gamma$, SAMHD1 degradation was blocked, (Fig. 1a, bottom row), even at high doses of VLPs-Vpx (Fig. 1b, dashed lines). Averaged over three independent experiments, IFN $\alpha$ or IFN $\gamma$ treatment blocked SAMHD1 degradation by fivefold (Fig. 1c). Therefore, we hypothesized the existence of one or several ISGs, induced by both IFN $\alpha$ and IFN $\gamma$, that protect SAMHD1 from degradation by Vpx-containing VLPs.

\section{A CRISPR screen identifies IFITMs as factors blocking Vpx-mediated degradation of SAMHD1}

IFN $\alpha$ could affect SAMHD1 degradation by Vpx by any of several mechanisms, such as blocking Vpx trafficking to the nucleus, post-translational modification of

\footnotetext{
(See figure on next page.)

Fig. 1 IFN treatment inhibits Vpx-mediated degradation of SAMHD1. THP1 cells were treated for $24 \mathrm{~h}$ with $1000 \mathrm{U} / \mathrm{mL}$ of IFNa, $1000 \mathrm{U} / \mathrm{mL}$ of IFNY or left untreated. The indicated amount of VLPS-Vpx pseudotyped with VSV-G (as determined by RT activity) was added to cells for 16 h: cells were then collected and SAMHD1 degradation was measured by flow cytometry. a Representative flow cytometry pots. $\mathbf{b}$ Representative dose response experiment. THP1 were treated with the indicated amount of VLPS-Vpx. The percentage of cells in which SAMHD1 is degraded was determined as shown in a. c Combined data from three independent experiments, using a viral dose within linear range. ${ }^{*} p<0.05$; ${ }^{* *} p<0.01$ ( $t$ test)
} 


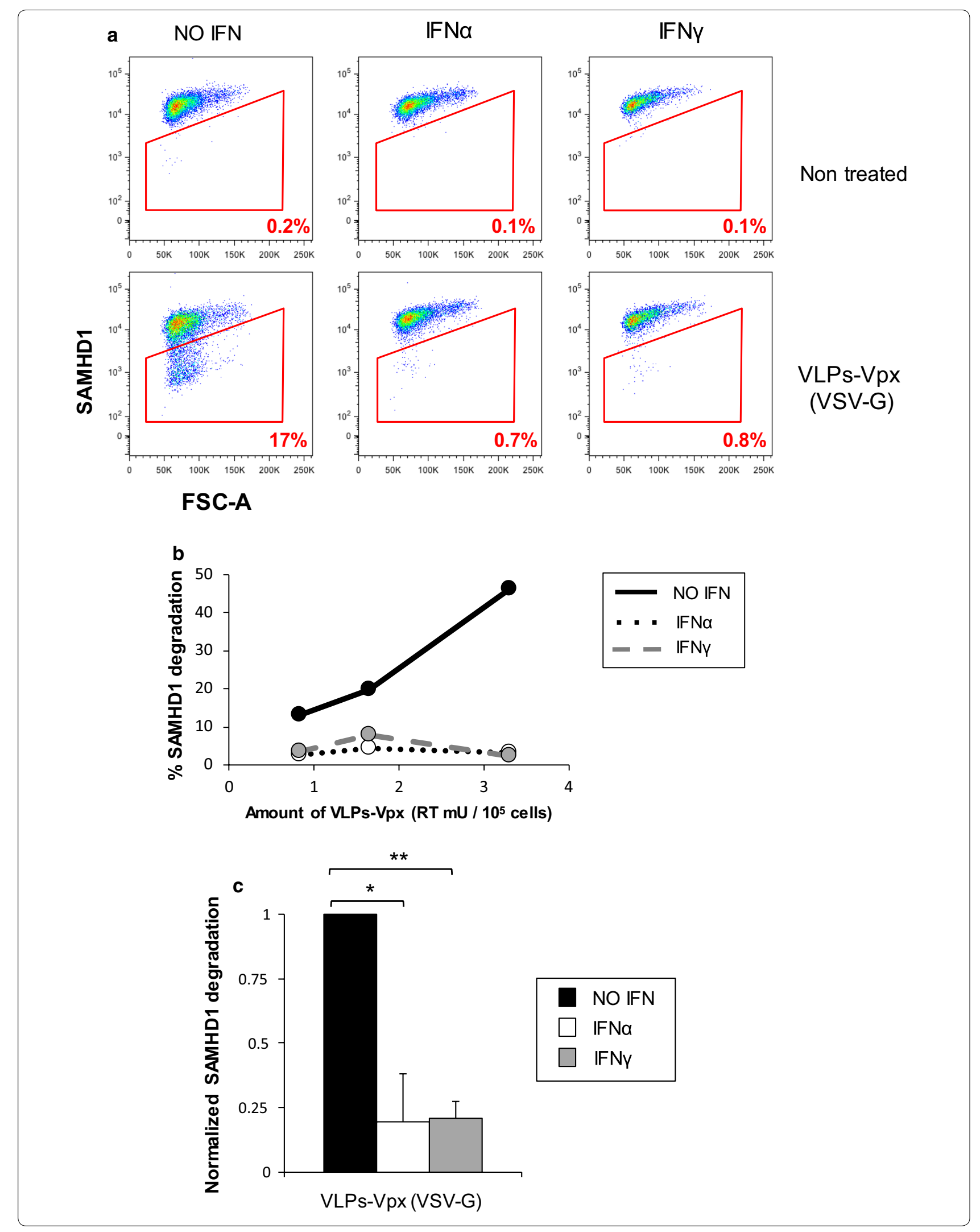


SAMHD1, interference with the degradation pathway through which Vpx targets SAMHD1, or, as described below, IFN $\alpha$ induction of genes affecting entry/fusion of the VLPs delivering Vpx. In order to identify the factor(s) responsible for this phenotype, we designed a CRISPR knockout screen taking advantage of the high-throughput qualities of both flow cytometry and next-generation sequencing technologies. We hypothesized that sgRNAs that target genes necessary for SAMHD1 protection from degradation would be enriched in the population of cells displaying low levels of SAMHD1. We first created a library of single-guide RNAs (sgRNAs) targeting 1906 human ISGs, with 8 different sgRNAs per gene and 200 non-targeting controls (NTCs) that do not target any loci in the human genome. We assembled sgRNAs into a lentiviral vector backbone that also encodes Cas9 and a puromycin resistance gene (OhAinle et al., manuscript in preparation). THP1 cells were transduced with this library, selected for puromycin resistance and cultured for 2 weeks to allow gene knockout to occur. The cells were treated with IFN $\alpha$, and incubated with VSV-G pseudotyped VLPs-Vpx as described in Fig. 1. Endogenous SAMHD1 levels were measured and cells were sorted using flow cytometry.

The gating strategy for sorting a pure population of SAMHD1 negative cells is outlined in Fig. 2a. First, we sorted cells based on their morphology to remove dead cells, debris and cell doublets, which may skew subsequent analyses. Non-viable cells that cannot be identified solely by their morphology were eliminated by incubation with a viability dye, in which they appeared high in the DAPI channel. Finally, cells were sorted based on their SAMHD1 levels. As expected, only a small fraction of cells (about 7\%) are SAMHD1 negative, which is consistent with data presented in Fig. 1 and with the hypothesis that only a very limited fraction of the CRISPR library will rescue SAMHD1 degradation. After sorting, we obtained $5 \times 10^{5}$ SAMHD1 negative cells and $3 \times 10^{6}$ SAMHD1 positive cells, which allows for a coverage of the library higher than 100X. The screen was performed with two technical replicates. After DNA extraction, sgRNA sequences in the different cell populations were amplified and deep-sequenced.

The frequency of each sgRNA within the SAMHD1 negative and positive populations was determined to calculate enrichment in the SAMHD1 negative population. sgRNAs enriched in the SAMHD1 negative fraction should target factors involved in IFN signaling, in SAMHD1 expression and stability, and may potentially target new ISGs protecting SAMHD1. In order to take into account results of all 8 sgRNAs targeting each gene in the library, we performed a gene-specific analysis with the MAGeCK tool that was developed for this purpose
[28]. This method assigns a score for each gene, factoring in the combined action of all sgRNAs, the enrichment of each sgRNA, and the biological replicate of the screen. Figure $2 \mathrm{~b}$ shows the top 20 genes from screen ranked by their-log10 MAGeCK score on the $\mathrm{X}$ axis. As validation of our strategy, at the very top of this list is SAMHD1 itself since sgRNAs targeting this gene should result in low protein levels of SAMHD1 (Fig. 2b, blue square). Factors necessary for IFN signaling such as the IFN receptor IFNAR1, the signaling molecules STAT1 and STAT2, and the transcription factor IRF9 are among the top scoring hits from the screen (Fig. 2b, orange triangles), further validating our strategy.

After these positive controls, IFITM3 is one of the highest hits together with poorly characterized genes such as COMMD3, EIF3L and SLC35A2. Of note, IFITM1 is also present in our list of top hits, although with a lower MAGeCK score (Fig. 2b) and IFITM2 ranks 232, with a much lower gene score (Additional file 1: Table S1). It should be pointed out, however, that because of the extensive homology between IFITMs (up to $90 \%$ at the nucleotide level), the sgRNAs used in our screen cannot completely distinguish between the different homologs. In particular, one sgRNA for IFITM1, which was much more enriched than the others, displayed perfect homology with IFITM3 (not shown).

In order to explain the IFN $\alpha$-induced protection of SAMHD1 phenotype, candidate hits should be significantly induced by IFN $\alpha$ treatment. Thus, we compared the gene scores from our screen to IFNa induction at the mRNA level in THP1 cells using a previously published dataset [29] (Fig. 2b, Y axis). While a number of our top hits, such as COMMD3, SLC35A2 and EIF3L, are reported to be poorly induced-if at all-at the transcriptional level by IFN $\alpha$ [29], IFITM proteins were highly induced by IFN $\alpha$ in THP1 cells [29], thus appearing to be the most likely candidate hits from this screen (Fig. 2b).

\section{Knockout of IFITM2/3 explains most of the phenotype}

To validate the results of our screen, we generated CRISPR knockout THP1 cells for specific IFITM proteins. Achieving knockout specificity for IFITMs is technically challenging, as previous studies have pointed out $[25,30]$, in particular for IFITM2 and IFITM3, which share $90 \%$ sequence identity at the nucleotide level. Therefore, we selected one sgRNA that specifically targets IFITM1 (12 and 13 nucleotide changes from IFITM2 and IFITM3, respectively) and one sgRNA that targets both IFITM2 and IFITM3 as it has perfect homology for both loci in THP1 cells, but differs by three nucleotides from IFITM1. A nontargeting control (NTC) sgRNA was included as a negative control. These sgRNAs were cloned into the 


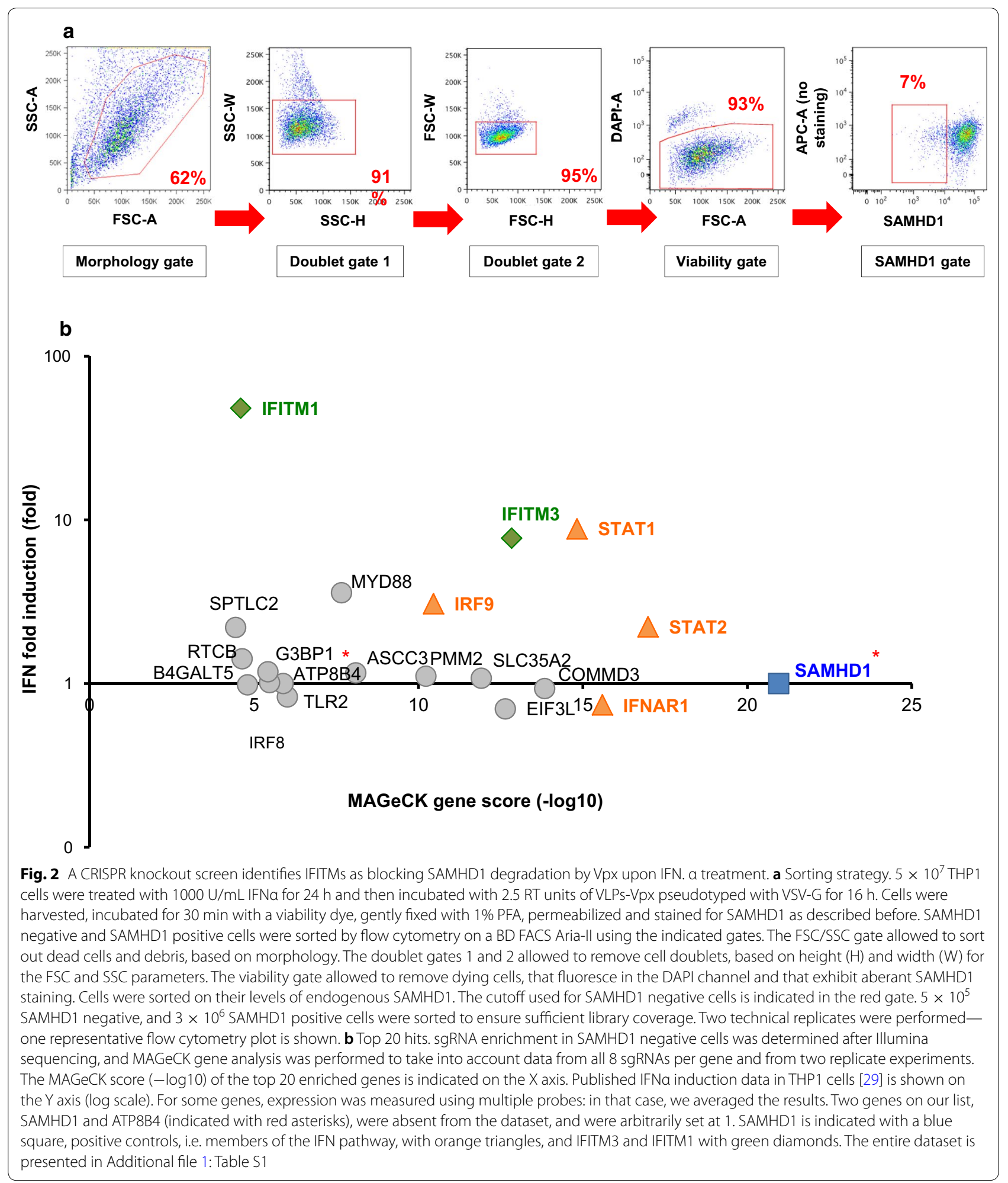

Cas9-expressing lentiviral vector, and THP1 cells were transduced and selected by puromycin treatment. We first attempted to generate clonal knockout cell lines, but heterogeneity of the cell line, a known issue with THP1 cells [31], caused significant clone-to-clone variability and complicated interpretation of the results 
(not shown). Instead, we used pools of knockout cells, in which, after puromycin selection, levels of IFITM expression were greatly reduced, as measured by Western Blot (Fig. 3a). We observed that the IFITM1 sgRNA appeared to be specific, as it knocked-out almost entirely the expression of IFITM1 without significantly altering levels of IFITM2 and IFITM3. The sgRNA targeting IFITM2/3 led to low levels of expression of both IFITM3 and IFITM2, even in presence of IFN $\alpha$, but left IFITM1 intact. Antibody specificity for IFITM2 vs IFITM3 is known to be difficult to achieve. Thus, to confirm our Western Blot results, we designed primers specific for the ifitm 2 and ifitm 3 loci (details in the Methods section), that were used for PCR amplification and sequencing of the CRISPR lesions present in THP1 knockout cells (Fig. 3b). Using TIDE analysis, a tool specifically designed to assess the percentage of cells edited by sgRNAs in heterogeneous populations [32], we determined that IFITM $2 / 3$ knockout cells are highly edited for ifitm 2 (71\%) and ifitm3 (78\%), while NTC and IFITM1 knockout cells showed no editing at these loci. These results confirm that the sgRNA has specificity for both IFITM2 and IFITM3, as the IFITM2/3 knockout cells are edited at both the ifitm 2 and ifitm 3 loci, but not at the ifitm 1 locus.

To ask if IFITM knockout rescues SAMHD1 degradation, knockout cell lines were treated with IFN $\alpha$ and with Vpx, as described previously, and SAMHD1 degradation was measured. We observed that knockout of IFITM2/3, but not IFITM1, led to a significant rescue of SAMHD1 degradation in presence of IFN $\alpha$. In the experiment presented in Fig. 3c, up to $34 \%$ of IFITM2/3 knockout cells (vs $<1 \%$ in NTC cells) had SAMHD1 degraded in the presence of IFNa. Averaged over three independent experiments, we observed that knocking out IFITM2/3 significantly rescued SAMHD1 degradation (Fig. 3d). These results suggest that IFITM3 and/or IFITM2, but not IFITM1, play a major role in inhibiting SAMHD1 degradation by VLPs-Vpx.

Since IFITM3 was the top ISG (apart from positive controls) enriched in our initial screen, we also generated a stable THP1 cell line over-expressing an HA-tagged version of IFITM3 (IFTIM3-HA), in which the levels of IFITM3 are within fivefold of those observed after IFN $\alpha$ treatment, as measured by Western Blot (Fig. 3e). We found that IFITM3-HA over-expression potently inhibited the ability of VSV-G pseudotyped VLPs-Vpx to degrade SAMHD1 in the absence of IFNa: averaged over 3 experiments, we observed a fourfold reduction in SAMHD1 degradation in IFITM3 expressing cells, compared to wild-type THP1 (Fig. 3f). The results of both knockout and over-expression experiments thus show that IFITM2/3 inhibit SAMHD1 degradation in THP1 cells.

\section{IFNa protection of SAMHD1 is envelope-dependent and affects entry of $\mathrm{Vpx}$ bearing VLPs into cells}

We hypothesized that IFITMs may act on SAMHD1 degradation by inhibiting VSV-G endosomal entry, as this envelope was used to pseudotype the Virus-Like Particles containing Vpx. Importantly, while IFITM3 restricts entry of VSV [6] and of lentiviral vectors pseudotyped with the VSV-G envelope [33], it has no effect on entry mediated by the Murine Leukemia Virus amphotropic envelope (A-MLV) [4]. Unlike VSV, MLV is thought to fuse mainly at the plasma membrane [34], although some reports suggest that it uses caveolae-mediated endocytosis $[35,36]$. Thus, we compared VLPs-Vpx pseudotyped with VSV-G, which had been used in Fig. 1 and in previous studies [10,12, 22, 27], with VLPs-Vpx pseudotyped with the Murine Leukemia Virus amphotropic envelope (A-MLV). We normalized the amounts of VLPs-Vpx used based on RT activity, and verified that the different VLPs packaged roughly similar levels of Vpx (Fig. 4a). When

\footnotetext{
(See figure on next page.)

Fig. 3 IFITMs block SAMHD1 degradation after treatment with VLPs-Vpx pseudotyped with VSV-G. a Knockout efficiency as measured by protein levels. THP1 were cells transduced with a lentivector coding for Cas9 and sgRNAs targeting either IFITM1 (IFITM1-KO, lanes 2 and 5) or IFITM2 and IFITM3 (IFITM2/3-KO, lanes 3 and 6) or encoding a non-targeting sgRNA (NTC, lanes 1 and 4). Cells were selected in puromycin for 2 weeks. Cells were treated for $24 \mathrm{~h}$ with 0 (lanes 1 through 3) or 1000 (lanes 4 through 6) U/mL IFNa and protein expression was measured by Western Blot. For IFNa treated cells, three times less lysate was loaded, in order to be able to visualize levels of IFITMs in the absence or presence of IFNa stimulation in the same exposure. Tubulin was used as a loading control. b Genomic DNA was extracted from THP1 NTC, IFITM1-KO or IFITM2/3-KO cells. The ifitm2 and ifitm 3 loci were amplified using specific PCR primers with a 3' mismatch. For each cell type, we verified that the PCR was specific by Sanger sequencing. The sequences at the two loci were compared to the reference sequence using TIDE analysis and the percentage of editing was quantified. c Cells were treated for $24 \mathrm{~h}$ with 0 or $1000 \mathrm{U} / \mathrm{mL}$ IFNa and incubated with the indicated amount of VLPs-Vpx pseudotyped with VSV-G. SAMHD1 degradation was measured by flow cytometry $16 \mathrm{~h}$ after treatment. One dose response experiment is shown. $\mathbf{d}$ Combined data from three independent experiments, using a viral dose within linear range. Each symbol represents an experiment. ${ }^{*} p<0.05$ ( $t$ test). e Overexpression of IFITM3 in THP1 cells was measured by Western Blot. Cells were lysed in NP40-DOC buffer and probed for IFITM3. Tubulin was used as a loading control. The band indicated with an asterisk corresponds to the HA tagged version of IFITM3. f SAMHD1 degradation assays. Cells were treated for $24 \mathrm{~h}$ with 0 or $1000 \mathrm{U} / \mathrm{mL}$ IFNa, then incubated with the indicated amount of VLPs-Vpx pseudotyped with VSV-G for $16 \mathrm{~h}$ and SAMHD1 degradation was measured by flow cytometry. Combined data from three independent experiments using a viral dose within linear range. ${ }^{*} p<0.05$ ( $t$ test)
} 


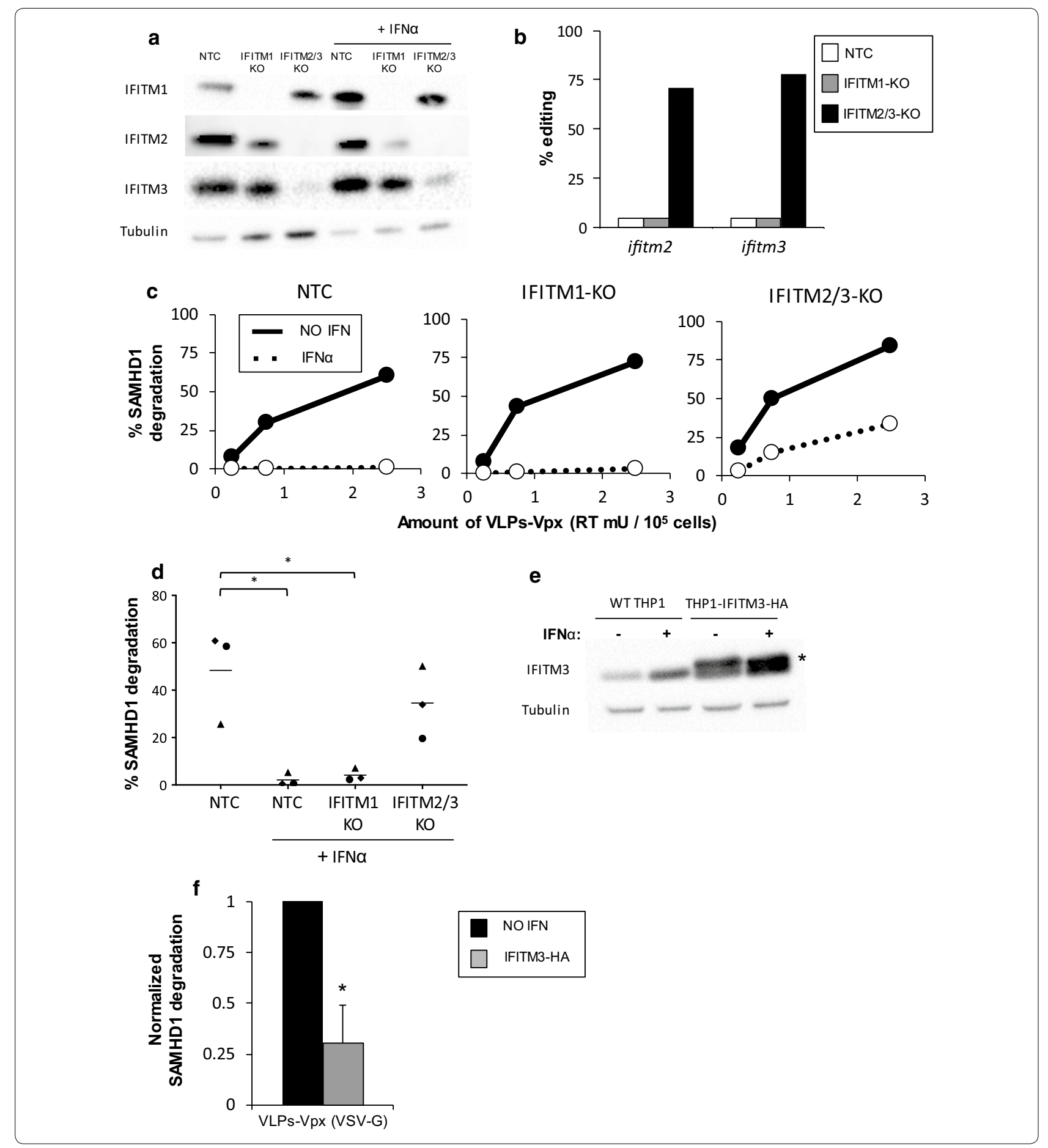

A-MLV was used instead of VSV-G, about 6 times more VLPs were required in order to achieve similar levels of SAMHD1 degradation, indicating that this envelope may be less fusogenic (Fig. 1b, 1.5 RT mU of VLPs VSV-G lead to $20 \%$ degradation compared to 9 RT mU of VLPs A-MLV, Fig. 4b). Strikingly, we observed that while
A-MLV env-pseudotyped Vpx-containing particles were able to cause degradation of SAMHD1 in the absence of IFN $\alpha$ (Fig. 4b, black lines), IFN $\alpha$ treatment had no effect on Vpx-mediated SAMHD1 degradation (Fig. 4b, dashed line). Averaged over three independent experiments, we found that there is no significant difference in SAMHD1 

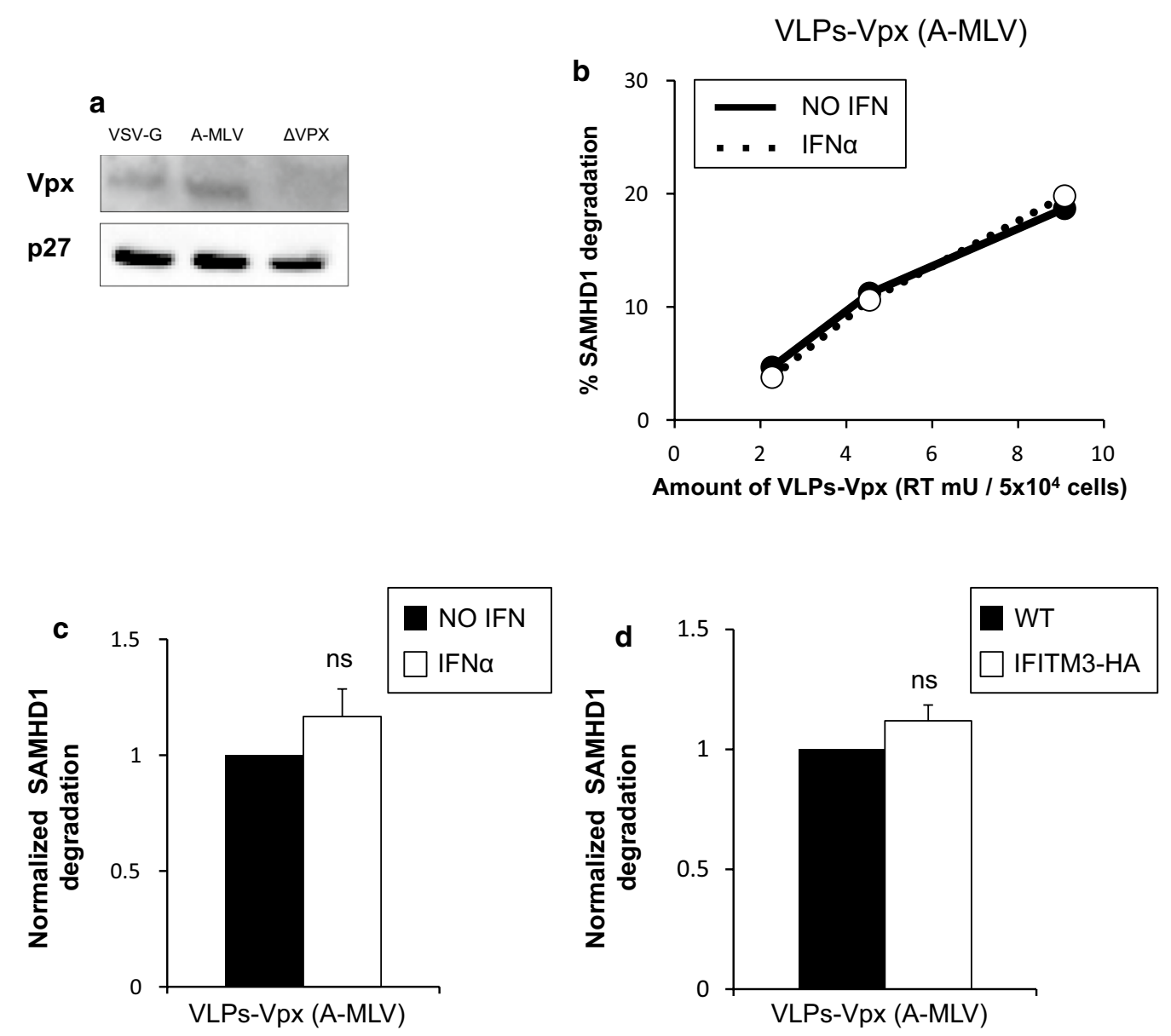

Fig. 4 IFN a does not block SAMHD1 degradation in THP1 cells when VLPS-Vpx are pseudotyped with A-MLV. a Levels of Vpx incorporation in VLPs pseudotyped with different envelopes. 250 RT mU of VLPs-Vpx pseudotyped with either VSV-G (left lane) or A-MLV (center lane) were loaded on a gel and levels of Vpx were probed by Western Blot. 250 RT mU of VLPs- $\triangle v p x$ pseudotyped with VSV-G (rigth lane) were used as a negative control. $50 \mathrm{RT} \mathrm{mU}$ of the same VLPs preparations were loaded and probed for p27 as a loading control. b THP1 cells were treated for $24 \mathrm{~h}$ with 0 (solid lines) or 1000 (dotted lines) U/mL of IFNa, then with the indicated amount of VLPs-Vpx pseudotyped with A-MLV (as determined by RT activity). Cells were treated with the indicated amounts of VLPs-Vpx and SAMHD1 degradation was measured by flow cytometry $16 \mathrm{~h}$ later. One representative experiment is shown. c Combined data from three independent experiments for the effect of IFNa (white bar), using a viral dose within linear range. $n=3$; ns non significant ( $t$ test). $\mathbf{d}$ Combined data from three independent experiments for the effect of IFITM3 overexpression (white bar), using a viral dose within linear range. $n=3$; $n$ s non significant ( $t$ test)

degradation by VLPs-Vpx particles pseudotyped with A-MLV (Fig. 4c), in contrast to VLPs-Vpx particles pseudotyped with VSV-G (Fig. 1c). IFITM3-HA over-expression also had no effect on SAMHD1 degradation when the A-MLV env was used (Fig. 4d), consistent with previous reports [4]. These results provide further evidence that, rather than directly acting on the SAMHD1/Vpx interface or on Vpx itself, IFN likely acts at the earlier stage of Vpx delivery by VLPs.

In order to initiate degradation of SAMHD1, Vpx must be released from incoming virions, enter the nucleus [12] and engage the DCAF4/DDB1/Cul4a complex $[15,16]$. Thus, monitoring SAMHD1 degradation is an indirect measure of the effects of IFN $\alpha$ and IFITMs on blocking release of viral components first into the cytoplasm. To show more directly that IFN $\alpha$ affects an early step of Vpx delivery into cells, we used the Vpr- $\beta$-lactamase (VprBlaM) assay, which measures entry of viral cores into the cytoplasm specifically, without taking into account capture of viral particles in endosomes.

We produced HIVAenv pseudotyped with VSV-G (HIV $\Delta e n v(\mathrm{VSV}-\mathrm{G})$ ) packaging Vpr-BlaM, and infected THP1 cells. Representative flow cytometry data and a dose response experiment are shown in Fig. 5a, b, respectively. IFITM3-HA over-expression significantly restricted VSV-G-mediated entry by about 


\section{(See figure on next page.)}

Fig. 5 IFN a treatment and IFITMs directly block viral fusion. a THP1 wild-type or overexpressing IFITM3-HA were treated for $24 \mathrm{~h}$ with 0 or 1000 $\mathrm{U} / \mathrm{mL}$ IFNa for $24 \mathrm{~h}$, and then infected with the indicated amount of HIV-1 $\triangle$ env(VSV-G) packaging the Vpr- $\beta$-lactamase fusion protein for $3 \mathrm{~h}$. Cells were then incubated with the fluorescent CCF2-AM substrate for $2 \mathrm{~h}$, fixed, and acquired by flow cytometry immediately. Representative flow cytometry plots are shown. $\mathbf{b}$ Representative dose response experiment. Solid black line: wild-type THP1 cells, dashed black line: wild-type THP1 treated with IFNa, grey dashed line: THP1 cells overexpressing IFITM3. c Combined data from three independent experiments, using a viral dose within linear range. ${ }^{* *} p<0.01$ (paired $t$ test). $\mathbf{d}$ THP1 cells wild-type (in black) or knockout for IFITM2/3 (in grey) were treated with IFNa (dashed lines) or untreated (solid lines) and infected with the indicated amount or HIV $\Delta$ enV(VSV-G). One representative dose response experiment is shown. e Combined data from four independent experiments, using a viral dose within linear range. Each symbol represents data from one experiment. ${ }^{* *} p<0.01$ (paired $t$ test)

threefold (Fig. 5c), consistent with its effect on Vpx delivery (Fig. 3b). IFN $\alpha$ had an even stronger effect, inhibiting VSV-G entry by about eightfold. This result further demonstrates that IFN $\alpha$ treatment and IFITM3 expression are able to restrict entry of viral particles pseudotyped with VSV-G, excluding other indirect effects on Vpx or SAMHD1. Similar to our results using SAMHD1 degradation experiments (Fig. 3), knocking-out IFITM2/3 rescued VSV-G fusion in the presence of IFN (Fig. 5d): the inhibition by IFN $\alpha$ decreased from roughly 30 -fold to 1.5-fold when IFITM2/3 were knocked-out even though we used pools of cells where the knockout efficiency was around 75\% (Fig. 3b). Averaged over four experiments, IFITM2/3 knockout significantly rescued entry of VSV-G pseudotyped particles into cells (Fig. 5e), demonstrating that IFITM2/3 indeed contribute to the IFN $\alpha$ entry block to VSV-G entry. Although we cannot exclude a role for other ISGs, we conclude that the main mechanism by which IFN $\alpha$ protects SAMHD1 from degradation is by inducing expression of IFITMs that block VSV-G-mediated entry of VLPs-Vpx.

\section{IFNa and IFITMs impose a stronger block on VSV-G-pseudotyped viruses than on wild-type HIV-1}

IFN $\alpha$ treatment, in part through the action of IFITMs, blocks entry of VSV-G pseudotyped viral particles. Because entry with the VSV-G envelope may differ from how HIV-1 enters cells we compared the magnitude of this block to the one imposed on HIV-1's own CXCR4tropic envelope. Importantly, these two envelopes have different mechanisms for viral entry: while VSV-Gmediated entry occurs through $\mathrm{pH}$-dependent fusion in the endosomes [37], productive entry of HIV-1 into its target cells likely happens by $\mathrm{pH}$-independent fusion at the plasma membrane, although this remains controversial $[38,39]$. Thus, we reasoned that the site of viral entry might dictate the sensitivity to IFN $\alpha$ and to IFITMs and affect lentiviruses bearing the HIV-1 or VSV-G envelopes differentially. To test this hypothesis, we directly compared the amount of IFN $\alpha$ block to HIV-1 that either contained its natural env or was pseudotyped with VSV-G in the entry assay described previously (Fig. 5).
Strikingly, while we observed stronger IFN $\alpha$ block on VSV-G-mediated entry than for HIV-1 env-mediated entry (24-fold vs 1.5-fold on average, respectively; Fig. 6a, b). Similarly, knockout of basal levels of IFITM2/3 (in absence of IFN $\alpha$ ) had a significant impact on VSVG-mediated entry, but much less on entry mediated by HIV-1 Env (fourfold vs 1.5-fold on average, Fig. 6c, d). In both cases, statistical analysis indicated that infection by VSV-G pseudotypes was significantly different than HIV-1 wt either in the presence of IFN $\alpha$ or in untreated cells lacking IFITM2 and IFITM3. These results suggest that VSV-G pseudotyping may expose the virus to a different subset of restriction factors that restrict viral fusion, including IFITMs, potentially by diverting HIV-1 from its natural entry pathway. These results are consistent with previous observations linking the route of HIV-1 entry and IFITM restriction [25].

To confirm that this difference in entry translates into different levels of infection in cell cultures, we performed single cycle infection assays. THP1 cells transduced with lentivectors encoding Non-Targeting Control (NTC) or IFITM2/3-targeting sgRNAs were infected with either HIV-1 1 env(VSV-G), which is only able to perform one cycle of infection, or with wild-type HIV-1. In order to ensure that the latter virus only performs one round of infection, the viral input was washed after $24 \mathrm{~h}$ and cells were treated with T-20, a peptide inhibiting membrane fusion of HIV-1 [40]. These experiments were performed in absence of IFN $\alpha$ with the rationale that other ISGs potently inhibiting HIV in THP1 cells, such as MxB could mask the effect of IFITMs [29]. Notably, IFITMs are significantly expressed at basal levels (Fig. 3a). Infection was monitored by measuring intracellular Gag levels $48 \mathrm{~h}$ post-infection. Consistent with our entry results, we observed that IFITM2/3 knockout greatly enhanced infection by HIV-1 $\operatorname{env}$ (VSV-G), but had a much less pronounced effect on wild-type HIV-1 infection (Fig. 6e, f). Taken together, these results suggest that basal levels of IFITM2 and IFITM3 expression inhibit VSV-G-mediated entry, but much less HIV-1 entry. Furthermore, we conclude that VSV-G-pseudotyping, although achieving higher rates of infection thanks to a higher envelope 


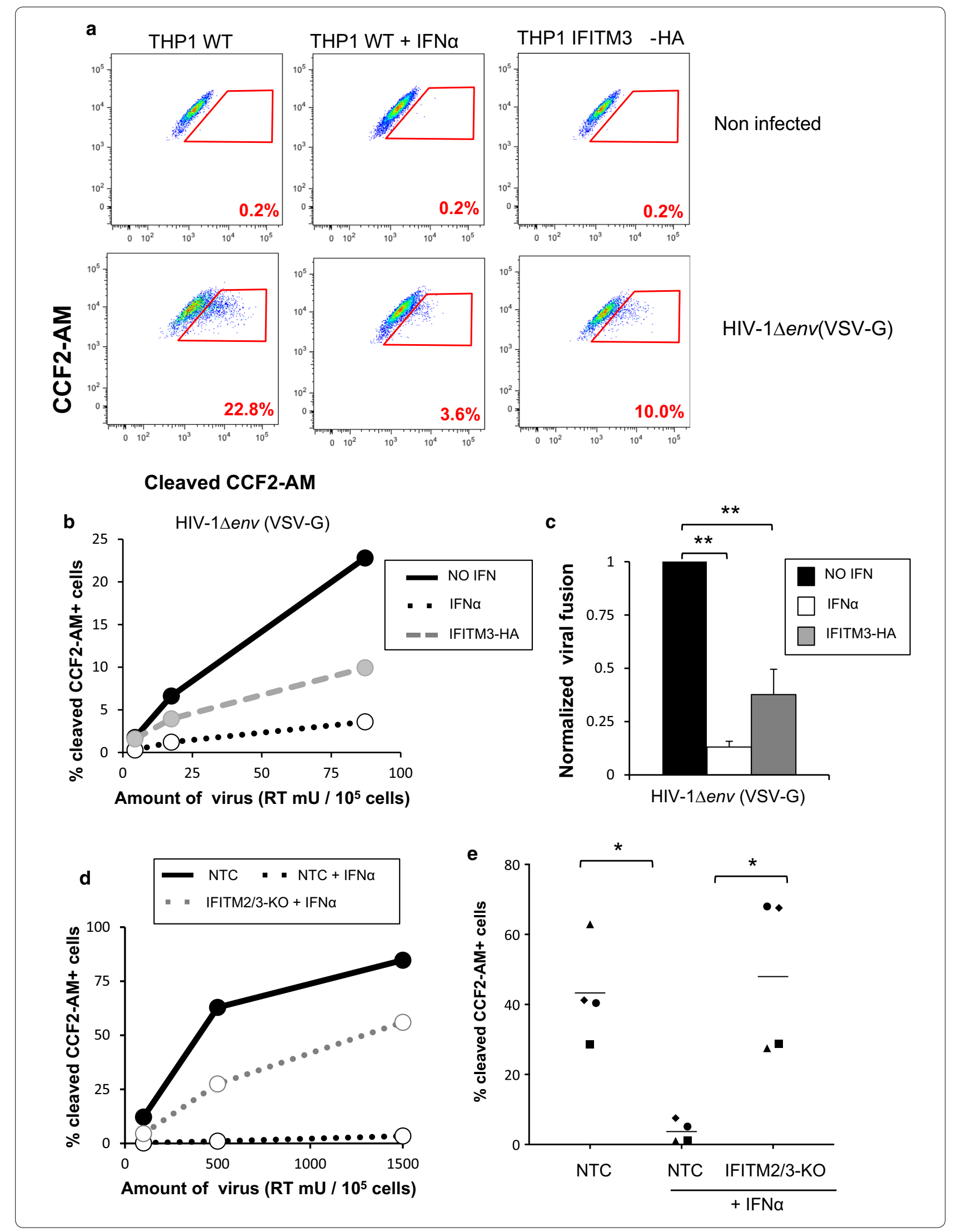




\begin{abstract}
(See figure on next page.)
Fig. 6 VSV-G pseudotyping leads to stronger restriction by IFNa and IFITM2/3. a, b THP1 wild-type or overexpressing IFITM3-HA were treated for

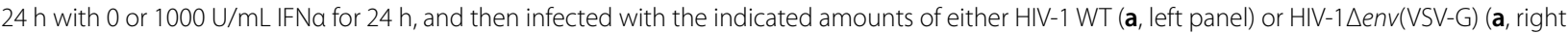
panel) packaging the Vpr- $\beta$-lactamase fusion protein for $3 \mathrm{~h}$. Cells were then incubated with the fluorescent CCF2-AM substrate for $2 \mathrm{~h}$, fixed, and acquired by flow cytometry immediately. a Representative dose response experiment. $\mathbf{b}$ Combined data from four independent experiments, using a viral dose within linear range. Each symbol representing data from one experiment. ${ }^{*} p<0.05$; ${ }^{* *} p<0.01$ ( $t$ test). $\mathbf{c}$, d THP1 cells transduced with a Non-Targeting Control (NTC) sgRNA or with an sgRNA targeting both IFITM2 and IFITM3 (IFITM2/3) were infected, in the absence of IFNa, with HIV WT (c, left panel) or HIV-1 $\triangle$ env(VSV-G) (c, right panel) packaging Vpr BlaM for $3 \mathrm{~h}$. Viral entry was measured as in panel a. $\mathbf{c}$ One representative dose response experiment. $\mathbf{d}$ Combined data from four independent experiments, using a viral dose within linear range. Each symbol represents one experiment. ${ }^{*} p<0.05$ ( $t$ test). e, f THP1 cells transduced with a non-targeting sgRNA (NTC) or with a sgRNA against IFITM2 and IFITM3 (IFITM2/3) were infected, in absence of IFNa, with HIVWT (e, left panel) or HIV-1 $\triangle$ env(VSV-G) (e, right panel). After $24 \mathrm{~h}$, viral input was washed, and cells were cultured in presence of $1 \mu \mathrm{M}$ of the entry inhibitor T-20, to prevent subsequence rounds of infection. The percentage of Gag positive cells were measured by flow cytometry at $48 \mathrm{~h}$ p.i. E: One representative dose response experiment. $\mathbf{f}$ Combined data from four independent experiments, using a viral dose within linear range. Each symbol representing data from one experiment. Each symbol represents one experiment. ${ }^{*} p<0.05$, ${ }^{* *} p<0.01$ ( $t$ test)
\end{abstract}

fusogenicity, changes the sensitivity of HIV-1 to an IFN $\alpha$ entry block by increasing sensitivity to IFITM-mediated restriction.

\section{Discussion}

A CRISPR screen for IFNa-induced factors that protect SAMHD1 from degradation

A flow cytometry-based CRISPR knockout screen approach identified IFITMs as being the most predominant ISGs underlying the protection of SAMHD1 from degradation after transduction with VSV-G-pseudotyped VLPs containing Vpx [22]. As the VSV-G used to pseudotype the VLPs delivering Vpx mediates entry into endosomes [37], where IFITMs (particularly IFITM3) are thought to act, our results argue that IFN $\alpha$ protects SAMHD1 from degradation by blocking viral entry of VLPs delivering Vpx into cells. The method described here that assays viral entry/fusion events by monitoring Vpx-mediated degradation of SAMHD1, could also be applied more generally to discover new genes affecting entry mediated by different envelope proteins.

\section{IFITMs directly contribute to the effect of IFNa on SAMHD1 degradation by VLPs-Vpx}

Because of high sequence homology, targeting IFITM3 without affecting levels of IFITM2 is challenging, as described in previous studies $[25,30]$. Thus, we could not formally demonstrate if IFITM2 or IFITM3 act synergistically, or alone in this phenotype. Nonetheless, we did show that IFITM3-HA overexpression is sufficient to inhibit degradation of SAMHD1 by VLPs-Vpx (Fig. 3). Although one previous study observed only a minor effect of IFN $\alpha$ treatment on viral entry of VSVG-pseudotyped lentivectors using the Vpr-BlaM assay [22], our eightfold IFN $\alpha$ block of VSV-G entry (Fig. 5), together with the fact that IFN $\alpha$ has no effect when an alternate delivery method was used (Fig. 4), strongly supports the idea that IFITMs block delivery of Vpx into the cytoplasm of IFN $\alpha$-treated cells. Therefore, the SAMHD1 protection phenotype described in the literature [22] can be explained by inhibition of Vpx delivery by IFITMs. Finally, other factors, such as COMMD3 or EIF3L also emerged as top hits from our screen (Fig. 2), and although they were minimally induced (if at all) by IFN $\alpha$ in THP1 cells, they may contribute to early events in VSV-G-mediated entry into cells.

\section{VSV-G pseudotyping changes HIV-1 sensitivity to IFNa and IFITMs}

In addition to blocking endosomal entry of VSV [6], IFITMs are also described to inhibit HIV-1 entry when expressed in target cells [41]. IFITM restriction in target cells may rely on a different mechanism than when IFITMs are expressed in producer cells. In addition to the role of IFITMs that are packaged into budding virions and restrict infection in target cells [33, 42, 43], IFITMs may, when expressed in target cells, also inhibit the hemifusion process [44, 45], and/or deregulate cholesterol homeostasis, resulting in reduced membrane fusion [46].

Changing the entry pathway used by HIV-1 by VSV-G pseudotyping directs membrane fusion to the endosomes instead of at the plasma membrane, and may influence HIV-1 sensitivity to ISGs, and to IFITMs in particular. Consistent with this idea, we observed that VSV-G pseudotypes are hyper sensitive to IFNo and IFITMs (Fig. 6). Moreover, the effect of IFITM3-HA on VSV-G-mediated entry did not completely recapitulate the magnitude of the IFN $\alpha$ block (Fig. 5), suggesting that other ISGs may play an additional role.

A recent report demonstrating that HIV-1 Transmitter/Founder strains are initially resistant to IFITM3, but become sensitive after viral escape from neutralizing antibodies [25]. Although it is important to note that the subcellular compartment in which HIV-1 fuses and enter 


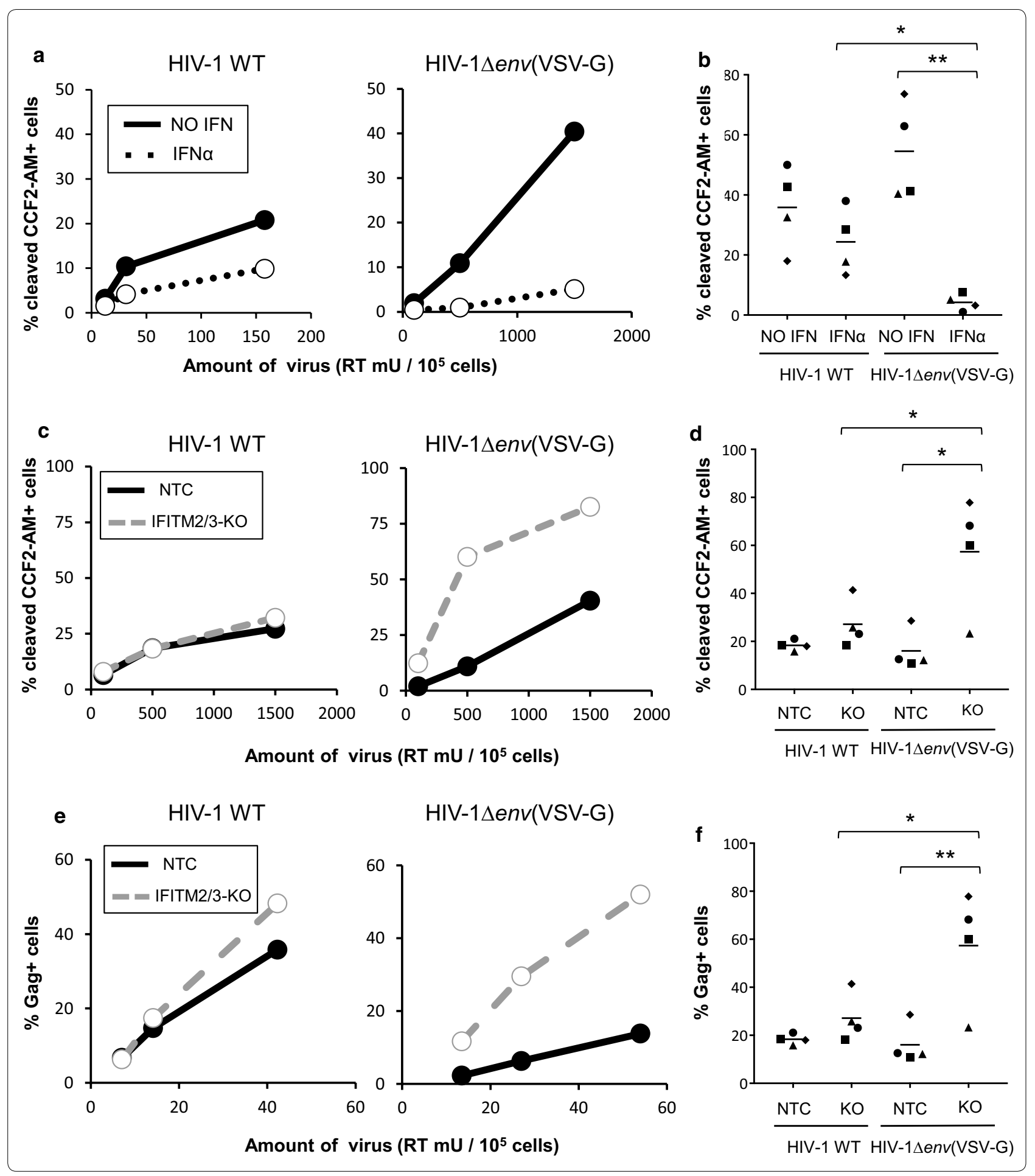

cells leading to productive infection remains controversial, and may depend on the cell type used [38, 39], one likely interpretation of our results is that in THP1 cells, HIV-1 fuses predominantly at the plasma membrane, rather than in endosomal vesicles where IFITM3 and other ISGs may act. VSV-G pseudotyping, by re-routing HIV-1 fusion to endosomes, may expose the virus to a different set of restriction factors. Our data suggest that HIV-1 strains that have evolved to fuse at the plasma membrane would avoid a potentially more restrictive 
environment imposed by IFITM2/3. The endosomal compartment may indeed represent a less productive route for HIV-1 entry, as has been suggested by a study in which a dominant negative variant of dynamin blocked endocytosis but had no effect on infection levels in $\mathrm{T}$ cells [47]. Importantly, VSV-G pseudotyping may not only affect viral entry, but also later steps in the viral life cycle, such as uncoating [48] or nuclear import and integration [49]. Thus, such considerations should be taken into account in studies that use VSV-G pseudotyping, as it may influence the antiviral factors that HIV-1 may be accessible to.

\section{Conclusions}

In this work, we used a CRISPR-Cas9 knockout flow cytometry screen to determine factor(s) are responsible for the block to SAMHD1 degradation in IFN $\alpha$ treated cells when Vpx is delivered via VSV-G pseudotyped viral particles. We identified IFITMs as the major factors involved in this phenomenon. Our results suggest that IFN $\alpha$ acts on Vpx delivery due to the effects of IFITMs on VSV-G-mediated entry/fusion rather than on the SAMHD1 degradation process itself. Indeed, we show that IFN $\alpha$ and IFITM2/3 have a much greater effect on entry of HIV-1 pseudotyped with VSV-G compared to HIV-1 bearing a CXCR4-tropic envelope. Thus, using VSV-G pseudotypes to study HIV restriction factors can be misleading because these pseudotypes may be exposed to antiviral factors that do not normally affect HIV-1. Finally, the CRISPR knockout screening approach we describe here could also be used to identify restriction factors specific for different viral envelopes using Vpx-mediated SAMHD1 degradation as an easy readout to screen for viral entry.

\section{Material and methods}

\section{Cells, plasmids and viruses}

THP1 cells were grown in RPMI 1640, supplemented with 10\% Fetal Bovine Serum (FBS) and penicillin/ streptomycin (100 $\mu \mathrm{g} / \mathrm{mL}$; Gibco \#15140-122). HEK293T cells were grown in Dulbecco modified Eagle medium (DMEM) supplemented with 10\% FBS and penicillin/streptomycin. The SIV $3+, \operatorname{SIV} 3 \Delta v p x$ and Vpr- $\beta$-lactamase plasmids are kind gifts from Olivier Schwartz [10, 11, 27, 50]. The pHIV-dTomato plasmid was obtained through Addgene (\#21374). The sgRNA library was synthesized in vitro (Twist Bioscience) and cloned in the lentiCRISPRv2 lentiviral backbone, and was a gift from Feng Zhang (Addgene plasmid \# 52961). pMD2.G and psPAX2 were gifts from Didier Trono (Addgene plasmids \#12259/12260). VLPs-Vpx were produced by transfecting HEK293T cells with SIV MAC Gag/ Pol (SIV3 + plasmid or SIV3 $\Delta v p x$ ) and VSV-G (pMD2.G plasmid) or A-MLV (A-MLV Env plasmid) at a 2.5: 1 ratio, and using $3 \mu \mathrm{L}$ of TransIT LT1 (\#MIR2305, Mirus) per $\mu \mathrm{g}$ of DNA. HIV-1 Vpr- $\beta$-lactamase viruses were produced by co-transfecting either HIV-1 proviral wild-type plasmid (pLAI) and Vpr- $\beta$-lactamase at a 3:1 ratio, or HIV-1 1 env (pLAI $\Delta e n v)$, VSV-G, and Vpr- $\beta$-lactamase at a 3:1:1 ratio. IFITM3-HA lentivector particles were made by co-transfecting the pHIV-dTomato-IFITM3-HA plasmid together with HIV-1 Gag/Pol (psPAX2 plasmid) and VSV-G at a 2:1.5:1 ratio. Lentiviral vector preparations containing the ISG library were made using the lentiCRISPRv2_ISG library assembly together with psPAX2 and VSV-G at a 2:1.5:1 ratio. For all virus and VLP production, supernatants were harvested $48-72 \mathrm{~h}$ post transfection, clarified by centrifugation, passed through a $0.22 \mu \mathrm{m}$ filter and concentrated by ultracentrifugation $(90 \mathrm{~min}$ at $90,000 \mathrm{~g})$. All transduction and infection experiments were carried out in presence of $20 \mu \mathrm{g} / \mathrm{mL}$ of DEAE-Dextran (Sigma; \# D9885). The following reagents were obtained through the NIH AIDS Reagent Program, Division of AIDS, NIAID, NIH: T-20,(Enfuvirtide); SVA-MLV-Env plasmid from Dr. Nathaniel Landau and Dr. Dan Littman [51].

\section{SAMHD1 degradation assay}

$5 \times 10^{4}$ THP1 cells were plated in 96-well plates and treated with $1000 \mathrm{U} / \mathrm{mL}$ universal type I IFN (\#112002 , PBL) or IFNY (\#11500-2, PBL) for $24 \mathrm{~h}$. The next day the cells were treated with the indicated amount of VLPsVpx for $16 \mathrm{~h}$. Cells were washed in PBS, and incubated for $15 \mathrm{~min}$ at room temperature with the Live/Dead Fixable Blue Dead Cell Stain Kit following instructions provided by the manufacturer (\#L34962, ThermoFisher), then fixed in $4 \%$ formaldehyde solution (diluted in PBS from a 37\% solution, Sigma \#252549) for $5 \mathrm{~min}$, and then permeabilized using PBS-Triton X-100 (Sigma, \#X100) $0.5 \%$ for $15 \mathrm{~min}$. Cells were then stained on ice for $30 \mathrm{~min}$ with anti-SAMHD1 antibody (clone I19-18, Millipore \#MABF933,) diluted to $0.5 \mu \mathrm{g} / \mathrm{mL}$ in PBS $1 \%$ $\mathrm{BSA}$, washed in PBS, and incubated on ice for $30 \mathrm{~min}$ with the Goat anti-mouse Alexa 488 secondary antibody (\#A-11001, Thermo Fisher), diluted to $4 \mu \mathrm{g} / \mathrm{mL}$ in PBS 1\% BSA. Flow cytometry data were acquired using a CANTO-II flow cytometer (BD).

\section{Flow cytometry CRISPR screen}

The screening experiment was performed with two technical replicates. $5 \times 10^{7}$ THP1 cells were transduced with lentiviral vectors containing the lentiCRISPRv2_ISG library. After 2 weeks of puromycin selection $(0.5 \mu \mathrm{g} / \mathrm{mL}$, \#P8833, Sigma), cells were treated with $1000 \mathrm{U} / \mathrm{mL}$ of universal type I IFN for $24 \mathrm{~h}$ and 
incubated with VLPs-Vpx for $16 \mathrm{~h}$. Cells were washed in PBS and incubated for $30 \mathrm{~min}$ at room temperature with the Live/Dead Fixable Blue Dead Cell Stain Kit following instructions provided by the manufacturer. Cells were washed in PBS and fixed with 1\% PFA for $15 \mathrm{~min}$. The fixation process was stopped by addition of 0.2 M Glycine. Cells were washed in PBS, and permeabilized using PBS/Triton X100 0.5\% for $15 \mathrm{~min}$. Cells were washed with PBS, stained on ice for $60 \mathrm{~min}$ with anti-SAMHD1 antibody diluted at $0.5 \mu \mathrm{g} / \mathrm{mL}$ in PBS 1\% BSA, washed in PBS, and incubated on ice for 45 min with the Goat anti-mouse Alexa 488 secondary antibody diluted to $4 \mu \mathrm{g} / \mathrm{mL}$ in PBS $1 \%$ BSA. Cells were washed and resuspended in sorting buffer (PBS 2\% FBS, $25 \mathrm{mM}$ Hepes, $5 \mathrm{mM}$ EDTA) and filtered. $5 \times 10^{5}$ SAMHD1 negative and $3 \times 10^{6}$ SAMHD1 positive cells were then sorted using an ARIA-II flow cytometer (BD), in polypropylene tubes previously coated with PBS 4\% BSA. Cells were pelleted and lysed in $300 \mu \mathrm{L}$ of chromatin immunoprecipitation buffer $(0.1 \%$ SDS, $10 \mathrm{mM}$ EDTA, $20 \mathrm{mM}$ EGTA, $300 \mathrm{mM} \mathrm{NaCl}, 10 \mathrm{mM}$ Tris $\mathrm{HCl} \mathrm{pH} \mathrm{8.1).} 3 \mu \mathrm{L}$ of proteinase $\mathrm{K}$ (\#19133, Qiagen) and $3 \mu \mathrm{L}$ of RNAse A (10 $\mu \mathrm{g} / \mathrm{mL}$, \#R4642-10MG, Sigma) were added to the lysates and cells were incubated at $65^{\circ} \mathrm{C}$ for $16 \mathrm{~h}$ to reverse crosslinking of protein and DNA. DNA was extracted using phenol chloroform (\#P2069-100 ML, Sigma), precipitated, washed in $70 \%$ ethanol, and resuspended in RNAse free water. sgRNAs were amplified from genomic DNA with a maximum of $2 \mu \mathrm{g}$ of template DNA per reaction using the Herculase II Fusion DNA Polymerase kit (Agilent, \#600679). A first PCR was carried out using the following primers: forward gagggcctatttcccatgattccttca and reverse ctgctgtccctgtaataaacccg. Round 1 PCR products were cleaned up using the QIAquick PCR purification kit (Qiagen, \#28106) and used as a template for the round 2 PCR, that allows barcoding and addition of the sequencing adapters, using a common reverse primer caagcagaagacggcatacgagatgtgactggagttcagacgtgtgctcttccgatcttgccactttttcaagttgataacggact coupled with a unique barcoding forward primer for each sample, here: F1 aatgatacggcgaccaccgagatctacactctttccctacacgacgctcttccgatctatctcgcgtacgtcttgtggaaaggacgaaacaccg and F2 aatgatacggcgaccaccgagatctacactctttccctacacgacgctcttccgatctactacagtgtcttgtggaaaggacgaaacaccg. PCR products were cleaned up and size selected using the Agencourt Ampure XP beads kit (Beckman Coulter, \# A63880), using a two-step purification with 1:1.2 and 1:1.5 DNA to beads ratios. Samples were quantified using the QUBIT dsDNA HS Assay Kit (Thermo Fisher Q32854), pooled at a $2 \mathrm{nM}$ concentration and submitted for sequencing on one HiSeq2500 (Illumina) lane, using the Rapid Mode (60 cycles).

\section{Statistical analysis}

sgRNA enrichment was analyzed using the Bioconductor package edgeR [52] and gene-level analysis was performed using MAGeCK [28]. The IFN $\alpha$ induction data was generated using a dataset (GSE46599) published by Goujon et al. [29] and re-analyzed using the lumi [53] and limma [54] Bioconductor packages. When several probes were used for a single genes, fold induction values were averaged.

\section{Reverse-transcriptase activity assay}

Viral stocks were quantified using an RT activity assay described before [55]. Briefly, viral supernatants were lysed in 2X lysis buffer $(0.25 \%$ Triton X-100, $50 \mathrm{mM}$ $\mathrm{KCl}, 100 \mathrm{mM}$ Tris $\mathrm{HCl}$, glycerol $40 \%$ ) in the presence of $4 \mathrm{U}$ RNAse inhibitor (Fermentas, \#EO0382). qRT-PCR reactions were prepared following instructions from the Takyon Rox SYBR MasterMix dTTP Blue kit (Eurogentec, \#UF-RSMT-B0101), with MS2 RNA used as a template (Roche, \#10165948001) and the following primers: tcctgctcaacttcctgtcgag and cacaggtcaaacctcctaggaatg. qRT-PCR was performed using a ABI QuantStudio5 Real Time PCR machine. Reactions were performed in duplicates, and titers were calculated using a standard curve made with a virus stock of previously determined RT units.

\section{Vpr- $\beta$-lactamase assays}

Viral entry was assayed with a protocol adapted from Cavrois et al. [50]. Briefly, indicated amounts of virus containing the $\mathrm{Vpr}-\beta$-lactamase fusion protein were used to infect $10^{5}$ THP1 cells. After $3 \mathrm{~h}$, cells were washed in cold $\mathrm{CO}_{2}$-independent media (Invitrogen), without $\mathrm{FBS}$, resuspended in $\mathrm{CO}_{2}$-independent media supplemented with $10 \%$ FBS, and incubated with the CCF2$\mathrm{AM}$ substrate following the instructions provided by the manufacturer (LiveBlazer FRET-B/G loading kit, \#K1023, Invitrogen), in the presence of $1.8 \mathrm{mM}$ Probenecid (\#P8761-25G, Sigma), for $2 \mathrm{~h}$ at room temperature in the dark. Cells were washed three times in cold $\mathrm{CO}_{2}$-independent media, then once in PBS, and finally fixed with $4 \%$ formaldehyde for $10 \mathrm{~min}$. Fluorescence of the CCF2-AM substrate was immediately measured by flow cytometry on a Canto-II (BD) using the AmCyan and Pacific-Blue channels.

\section{Generation of cell lines overexpressing IFITMs or with IFITM knockout}

RNA was extracted from $10^{6}$ THP1 cells using the Rneasy plus mini Kit (\#74134, Qiagen). RT-PCR was performed to amplify IFITM3 cDNA and add a C-terminal HA-tag and restriction sites using the SuperScript ${ }^{\circledR}$ III One-Step RT-PCR System with Platinum Taq DNA Polymerase 
kit (\#12574018, Invitrogen) and the following primers: gatctctagaatcgatatgaatcacactgtccaaacct and gatcggatccggtaccctaagcgtaatctggaacatcgtatgggtatccataggcctggaagatca. IFITM3-HA was then cloned into the pHIV-dTomato backbone using the $\mathrm{XbaI} / \mathrm{BamHI}$ restriction sites, and clones were verified by sequencing. THP1 cells were spinoculated with lentivectors encoding IFITM3-HA, and overexpression was verified by monitoring dTomato levels. Cells were harvested and lysed in the NP40-DOC lysis buffer (NP40 1\%, deoxycholate $0.2 \%, \mathrm{NaCl} 120 \mathrm{mM}$, Tris $20 \mathrm{mM}$ ) and IFITM3 levels were also monitored by Western Blot analysis (\#11714-1-AP, Proteintech; 1/1000). sgRNAs targeting IFITM1 or IFITM2/3 were cloned in the lentiCRISPRv2 backbone by BsmBI restriction of the backbone, annealing of sense and antisense oligos $\left(5 \mathrm{~min}\right.$ at $95^{\circ} \mathrm{C}$ ) and ligation. The following oligos were used (the $20 \mathrm{bp}$-gene targeting sequence in all caps): IFITM1 sense caccgCAGAGCCGAATACCAGTAAC and antisense aaacGTTACT GGTATTCGGCTCTGc; IFITM2/3 sense caccgGTGGATCACGGTGGACGTCG and antisense aaacCGACGTCCACCGTGATCCACc; NTC sense caccgACGGAGGCTAAGCGTCGCAA and antisense aaacTTGCGACGCTTAGCCTCCGTc. Lentiviral particles were produced as indicated before and used to transduce THP1 cells. After 1-2 weeks of puromycin selection (1 $\mu \mathrm{g} / \mathrm{mL}$, \#P8833, Sigma), genomic DNA was extracted using the QuickExtract kit (Lucigen, QE09050) by resuspending cells in $100 \mu \mathrm{L}$ of the solution, and by denaturing for $20 \mathrm{~min}$ at $60^{\circ} \mathrm{C}$ and $20 \mathrm{~min}$ at $95^{\circ} \mathrm{C}$. The following primers, specific for each ifitm locus due to a mismatch at the 3 ' nucleotide, were used for locus specific amplification: IFITM2-F aagaggaaactgttgagaaaacgg, IFITM2-R cgtgtgaggataaagggctgatg, IFITM3-F accatcccagtaacccgaccg, IFITM3-R gctgatacaggactcggctcc. Amplicons were sequenced and the percentage of editing was quantified using TIDE analysis [32] (https://tidecalculator.nki.nl/). Knockout was also verified by Western Blot using NP40-DOC as the lysis buffer and antibodies specific for IFITM1 (\#60074-1-Ig, Proteintech; 1/1000), IFITM2 (\#66137-1-Ig, Proteintech; 1/1000), IFITM3 (\#11714-1-AP, Proteintech; 1/1000).

\section{Single cycle infection assays}

$10^{5}$ cells were infected with HIV-1 WT or HIV$1 \Delta e n v(\mathrm{VSV}-\mathrm{G})$ with the indicated amounts of virus. After $24 \mathrm{~h}$, cells were washed in PBS and the T-20 entry inhibitor (NIH AIDS reagent program, \#12732) was added at a $1 \mu \mathrm{M}$ concentration to prevent subsequent rounds of infection. Intracellular Gag levels were measured by flow cytometry $36 \mathrm{~h}$ post infection using KC57-FITC (Beckman Coulter \#664665).

\section{Additional file}

Additional file 1: Table S1. Complete MAGeCK analysis. For each gene present in the knockout ISG library, we indicate the MAGeCK gene score, rank, and the associated $p$ value as determined by MAGeCK. Values of IFNa induction based on Goujon et al. [29] are also shown

\section{Authors' contributions}

FR designed and performed experiments, and analyzed results. MO helped perform experiments. ME helped design experiments and analyzed results. All authors wrote the manuscript and read and approved the final version.

\section{Acknowledgements}

We thank Olivier Schwartz for sharing reagents. We thank Loïc Dragin and Florence Margottin-Goguet for helpful discussion and Nicholas Chesarino and Amit Sharma for critical reading of the manuscript. We thank the Fred Hutch Flow Cytometry and Genomics Shared Resources. We thank Ryan Basom for help in data analysis.

Competing interests

The authors declare that they have no competing interests.

Ethics approval and consent to participate

Not applicable.

\section{Funding}

This work was supported by CCEH Pilot Grant P30 DK56465 to MO and R01 Al30927 and R01 GM11570 to ME. The funders had no role in study design, data collection and interpretation, or the decision to submit the work for publication.

\section{Publisher's Note}

Springer Nature remains neutral with regard to jurisdictional claims in published maps and institutional affiliations.

Received: 12 January 2018 Accepted: 13 March 2018

Published online: 20 March 2018

\section{References}

1. Schoggins JW, Rice CM. Interferon-stimulated genes and their antiviral effector functions. Curr Opin Virol. 2011;1:519-25.

2. Bailey CC, Zhong G, Huang IC, Farzan M. IFITM-family proteins: the cell's first line of antiviral defense. Annu Rev Virol. 2014;1:261-83.

3. Weston S, Czieso S, White IJ, Smith SE, Wash RS, Diaz-Soria C, et al. Alphavirus restriction by IFITM proteins. Traffic. 2016;17:997-1013.

4. Brass AL, Huang IC, Benita Y, John SP, Krishnan MN, Feeley EM, et al. The IFITM proteins mediate cellular resistance to influenza A H1N1 virus, West Nile virus, and dengue virus. Cell. 2009;139:1243-54.

5. Narayana SK, Helbig KJ, McCartney EM, Eyre NS, Bull RA, Eltahla A, et al. The interferon-induced transmembrane proteins, IFITM1, IFITM2, and IFITM3 inhibit hepatitis C virus entry. J Biol Chem. 2015;290:25946-59.

6. Weidner JM, Jiang D, Pan XB, Chang J, Block TM, Guo JT. Interferoninduced cell membrane proteins, IFITM3 and tetherin, inhibit vesicular stomatitis virus infection via distinct mechanisms. J Virol. 2010;84:12646-57.

7. Ambrose Z, Aiken C. HIV-1 uncoating: connection to nuclear entry and regulation by host proteins. Virology. 2014.

8. Accola MA, Bukovsky AA, Jones MS, Gottlinger HG. A conserved dileucine-containing motif in p6(gag) governs the particle association of Vpx and Vpr of simian immunodeficiency viruses SIV(mac) and SIV(agm). J Virol. 1999;73:9992-9.

9. Jauregui P, Logue EC, Schultz ML, Fung S, Landau NR. Degradation of SAMHD1 by Vpx Is independent of uncoating. J Virol. 2015;89:5701-13. 
10. Laguette N, Sobhian B, Casartelli N, Ringeard M, Chable-Bessia C, Segeral $\mathrm{E}$, et al. SAMHD1 is the dendritic- and myeloid-cell-specific HIV-1 restriction factor counteracted by Vpx. Nature. 2011;474:654-7.

11. Hrecka K, Hao C, Gierszewska M, Swanson SK, Kesik-Brodacka M, Srivastava S, et al. Vpx relieves inhibition of HIV-1 infection of macrophages mediated by the SAMHD1 protein. Nature. 2011;474:658-61.

12. Lahouassa H, Daddacha W, Hofmann H, Ayinde D, Logue EC, Dragin L, et al. SAMHD1 restricts the replication of human immunodeficiency virus type 1 by depleting the intracellular pool of deoxynucleoside triphosphates. Nat Immunol. 2012;13:223-8.

13. Descours B, Cribier A, Chable-Bessia C, Ayinde D, Rice G, Crow Y, et al. SAMHD1 restricts HIV-1 reverse transcription in quiescent CD4(+) T-cells. Retrovirology. 2012;9:87.

14. Lim ES, Fregoso OI, McCoy CO, Matsen FA, Malik HS, Emerman M. The ability of primate lentiviruses to degrade the monocyte restriction factor SAMHD1 preceded the birth of the viral accessory protein Vpx. Cell Host Microbe. 2012;11:194-204.

15. Sharova N, Wu Y, Zhu X, Stranska R, Kaushik R, Sharkey M, et al. Primate lentiviral Vpx commandeers DDB1 to counteract a macrophage restriction. PLoS Pathog. 2008:4:e1000057.

16. Ahn J, Hao C, Yan J, DeLucia M, Mehrens J, Wang C, et al. HIV/simian immunodeficiency virus (SIV) accessory virulence factor Vpx loads the host cell restriction factor SAMHD1 onto the E3 ubiquitin ligase complex CRL4DCAF1. J Biol Chem. 2012;287:12550-8.

17. Fregoso Ol, Ahn J, Wang C, Mehrens J, Skowronski J, Emerman M. Evolutionary toggling of $V p x / N p$ r specificity results in divergent recognition of the restriction factor SAMHD1. PLoS Pathog. 2013;9:e1003496.

18. Cribier A, Descours B, Valadao AL, Laguette N, Benkirane M. Phosphorylation of SAMHD1 by cyclin A2/CDK1 regulates its restriction activity toward HIV-1. Cell Rep. 2013;3:1036-43.

19. Bonifati S, Daly MB, St Gelais C, Kim SH, Hollenbaugh JA, Shepard C, et al. SAMHD1 controls cell cycle status, apoptosis and HIV-1 infection in monocytic THP-1 cells. Virology. 2016:495:92-100.

20. Yan J, Hao C, DeLucia M, Swanson S, Florens L, Washburn MP, et al. CyclinA2-cyclin-dependent kinase regulates SAMHD1 protein phosphohydrolase domain. J Biol Chem. 2015;290:13279-92.

21. Kudoh A, Miyakawa K, Matsunaga S, Matsushima Y, Kosugi I, Kimura H, et al. H11/HSPB8 restricts HIV-2 Vpx to restore the anti-viral activity of SAMHD1. Front Microbiol. 2016;7:883.

22. Dragin L, Nguyen LA, Lahouassa H, Sourisce A, Kim B, Ramirez BC, et al. Interferon block to HIV-1 transduction in macrophages despite SAMHD1 degradation and high deoxynucleoside triphosphates supply. Retrovirology. 2013;10:30.

23. Riess M, Fuchs NV, Idica A, Hamdorf M, Flory E, Pedersen IM, et al. Interferons induce expression of SAMHD1 in monocytes through downregulation of miR-181a and miR-30a. J Biol Chem. 2017;292:264-77.

24. Yang $S$, Zhan $Y$, Zhou $Y$, Jiang $Y$, Zheng $X$, Yu L, et al. Interferon regulatory factor 3 is a key regulation factor for inducing the expression of SAMHD1 in antiviral innate immunity. Sci Rep. 2016;6:29665.

25. Foster TL, Wilson H, Iyer SS, Coss K, Doores K, Smith S, et al. Resistance of transmitted founder HIV-1 to IFITM-mediated restriction. Cell Host Microbe. 2016;20:429-42.

26. Goujon C, Jarrosson-Wuilleme L, Bernaud J, Rigal D, Darlix JL, Cimarelli A With a little help from a friend: increasing HIV transduction of monocytederived dendritic cells with virion-like particles of SIV(MAC). Gene Ther. 2006;13:991-4.

27. Berger G, Goujon C, Darlix JL, Cimarelli A. SIVMAC Vpx improves the transduction of dendritic cells with nonintegrative HIV-1-derived vectors. Gene Ther. 2009;16:159-63.

28. Li W, Xu H, Xiao T, Cong L, Love Ml, Zhang F, et al. MAGeCK enables robust identification of essential genes from genome-scale CRISPR/Cas9 knockout screens. Genome Biol. 2014:15:554.

29. Goujon C, Moncorge O, Bauby H, Doyle T, Ward CC, Schaller T, et al. Human MX2 is an interferon-induced post-entry inhibitor of HIV-1 infection. Nature. 2013;502:559-62.

30. Wu X, Dao Thi VL, Huang Y, Billerbeck E, Saha D, Hoffmann HH, et al. Intrinsic immunity shapes viral resistance of stem cells. Cell. 2017.

31. Aldo PB, Craveiro V, Guller S, Mor G. Effect of culture conditions on the phenotype of THP-1 monocyte cell line. Am J Reprod Immunol. 2013:70:80-6
32. Brinkman EK, Chen T, Amendola M, van Steensel B. Easy quantitative assessment of genome editing by sequence trace decomposition. Nucleic Acids Res. 2014:42:e168.

33. Tartour K, Appourchaux R, Gaillard J, Nguyen XN, Durand S, Turpin J, et al. IFITM proteins are incorporated onto HIV-1 virion particles and negatively imprint their infectivity. Retrovirology. 2014;11:103.

34. McClure MO, Sommerfelt MA, Marsh M, Weiss RA. The $\mathrm{pH}$ independence of mammalian retrovirus infection. J Gen Virol. 1990;71(Pt 4):767-73.

35. Beer C, Andersen DS, Rojek A, Pedersen L. Caveola-dependent endocytic entry of amphotropic murine leukemia virus. J Virol. 2005;79:10776-87.

36. Katen LJ, Januszeski MM, Anderson WF, Hasenkrug KJ, Evans LH. Infectious entry by amphotropic as well as ecotropic murine leukemia viruses occurs through an endocytic pathway. J Virol. 2001;75:5018-26.

37. Sun X, Yau VK, Briggs BJ, Whittaker GR. Role of clathrin-mediated endocytosis during vesicular stomatitis virus entry into host cells. Virology. 2005:338:53-60.

38. Marin M, Melikyan GB. Can HIV-1 entry sites be deduced by comparing bulk endocytosis to functional readouts for viral fusion? J Virol. 2015;89:2985

39. Herold N, Muller B, Krausslich HG. Reply to "Can HIV-1 entry sites be deduced by comparing bulk endocytosis to functional readouts for viral fusion?". J Virol. 2015;89:2986-7.

40. Kilby JM, Hopkins S, Venetta TM, DiMassimo B, Cloud GA, Lee JY, et al. Potent suppression of HIV-1 replication in humans by T-20, a peptide inhibitor of gp41-mediated virus entry. Nat Med. 1998:4:1302-7.

41. Lu J, Pan Q, Rong L, He W, Liu SL, Liang C. The IFITM proteins inhibit HIV-1 infection. J Virol. 2011:85:2126-37.

42. Compton AA, Bruel T, Porrot F, Mallet A, Sachse M, Euvrard M, et al. IFITM proteins incorporated into HIV-1 virions impair viral fusion and spread. Cell Host Microbe. 2014;16:736-47.

43. Yu J, Li M, Wilkins J, Ding S, Swartz TH, Esposito AM, et al. IFITM proteins restrict HIV-1 infection by antagonizing the envelope glycoprotein. Cell Rep. 2015;13:145-56.

44. Li K, Markosyan RM, Zheng YM, Golfetto O, Bungart B, Li M, et al. IFITM proteins restrict viral membrane hemifusion. PLoS Pathog. 2013:9:e1003124

45. Desai TM, Marin M, Chin CR, Savidis G, Brass AL, Melikyan GB. IFITM3 restricts influenza $A$ virus entry by blocking the formation of fusion pores following virus-endosome hemifusion. PLOS ONE. 2014;10:e1004048.

46. Amini-Bavil-Olyaee S, Choi YJ, Lee JH, Shi M, Huang IC, Farzan M, et al. The antiviral effector IFITM3 disrupts intracellular cholesterol homeostasis to block viral entry. Cell Host Microbe. 2013:13:452-64.

47. Herold N, Anders-Osswein M, Glass B, Eckhardt M, Muller B, Krausslich HG. HIV-1 entry in SupT1-R5, CEM-ss, and primary CD4 + T cells occurs at the plasma membrane and does not require endocytosis. J Virol. 2014;88:13956-70.

48. Brun S, Solignat M, Gay B, Bernard E, Chaloin L, Fenard D, et al. VSV-G pseudotyping rescues HIV-1 CA mutations that impair core assembly or stability. Retrovirology. 2008;5:57.

49. Yu D, Wang W, Yoder A, Spear M, Wu Y. The HIV envelope but not VSV glycoprotein is capable of mediating HIV latent infection of resting CD4 T cells. PLoS Pathog. 2009;5:e1000633.

50. Cavrois M, De Noronha C, Greene WC. A sensitive and specific enzymebased assay detecting HIV-1 virion fusion in primary T lymphocytes. Nat Biotechnol. 2002;20:1151-4

51. Landau NR, Page KA, Littman DR. Pseudotyping with human T-cell leukemia virus type I broadens the human immunodeficiency virus host range. J Virol. 1991;65:162-9.

52. Robinson MD, McCarthy DJ, Smyth GK. edgeR: a Bioconductor package for differential expression analysis of digital gene expression data. Bioinformatics. 2010;26:139-40.

53. Du P, Kibbe WA, Lin SM. lumi: a pipeline for processing Illumina microarray. Bioinformatics. 2008;24:1547-8.

54. Wettenhall JM, Smyth GK. limmaGUI: a graphical user interface for linear modeling of microarray data. Bioinformatics. 2004;20:3705-6.

55. Vermeire J, Naessens $E$, Vanderstraeten $H$, Landi A, lannucci $V$, Van Nuffel $A$, et al. Quantification of reverse transcriptase activity by real-time PCR as a fast and accurate method for titration of HIV, lenti- and retroviral vectors. PLoS ONE. 2012;7:e50859. 